\title{
Corela
}

Cognition, représentation, langage

HS-21 | 2017

Linguistique de corpus : vues sur la constitution, l'analyse et l'outillage

\section{Pièges méthodologiques des corpus parallèles et comment les éviter}

\section{Olga Nádvorníková}

\section{OpenEdition}

Journals

Édition électronique

URL : http://journals.openedition.org/corela/4810

DOI : 10.4000/corela.4810

ISSN : 1638-573X

Éditeur

Cercle linguistique du Centre et de l'Ouest - CerLICO

Référence électronique

Olga Nádvorníková, «Pièges méthodologiques des corpus parallèles et comment les éviter », Corela [En ligne], HS-21 | 2017, mis en ligne le 20 février 2017, consulté le 30 avril 2019. URL : http:// journals.openedition.org/corela/4810; DOI : 10.4000/corela.4810

Ce document a été généré automatiquement le 30 avril 2019

\section{(c) (i) (8)}

Corela - cognition, représentation, langage est mis à disposition selon les termes de la licence Creative Commons Attribution - Pas d'Utilisation Commerciale - Partage dans les Mêmes Conditions 4.0 International. 


\title{
Pièges méthodologiques des corpus parallèles et comment les éviter
}

\author{
Olga Nádvorníková
}

\section{Introduction}

L'objectif de cet article ${ }^{1}$ est de présenter certains problèmes méthodologiques liés à l'exploitation de corpus parallèles, c'est-à-dire de corpus composés de textes originaux et de leurs traductions respectives, et de proposer des principes et des règles d'utilisation qui pourraient permettre de les résoudre ${ }^{2}$.

De nos jours, il existe un grand nombre de corpus parallèles, couvrant des langues très variées : non seulement l'anglais et le français (Hansard, cf. Roukos - Graff - Melamed 1995), mais aussi des langues slaves ( arasol $^{3}$ ) ou scandinaves, telles que le norvégien et le suédois (Johansson - Hofland 1994), etc ${ }^{4}$. Ces corpus varient en ce qui concerne le nombre des langues impliquées (une paire de langues vs corpus multilingues ${ }^{5}$ ), les types de textes contenus (littéraires, juridiques, journalistiques, scientifiques, etc.), le type d'annotation ${ }^{6}$, le niveau d'alignement des versions parallèles (en général au niveau des phrases) ou l'accessibilité. L'exemple suivant est tiré du corpus multilingue InterCorp ( www.korpus.cz/intercorp) ${ }^{7}$, sur lequel nous allons illustrer les principes méthodologiques de l'utilisation de corpus parallèles :

[FR] «Moi, se dit le petit prince, si j'avais cinquante-trois minutes à dépenser, je marcherais tout doucement vers une fontaine ... " (Antoine de Saint-Exupéry, Le Petit prince, 1999/1943)

[ALL] "Wenn ich dreiundfünfzig Minuten übrig hätte", sagte der kleine Prinz," würde ich ganz gemächlich zu einem Brunnen laufen..." (trad. par Leitgeb, Grete ; Leitgeb, Josef, 1956)

[ANG] "As for me," said the little prince to himself, "if I had fifty-three minutes to spend as I liked, I should walk at my leisure toward a spring of fresh water." (trad. par Katherine Woods, 1971)

[CS] Kdybych já měl padesát tři minuty nazbyt, řekl si malý princ, šel bych docela pomaloučku ke studánce... (trad. par Zdeňka Stavinohová, 1989) 
[HIN] "अगर मेरे पास तिरपन मिनट बिताने को होते " छोटे राजकुमार ने सोचा,' तो मैं धीरे - धीरे एक जलाशय की ओर चल पड़ता ... ।" (trad. par किशोर बलवीर, जगवंश, 2009)

[IT] "Io", disse il piccolo principe, "se avessi cinquantatré minuti da spendere, camminerei adagio adagio verso una fontana..." (trad. par Nini Bompiani Bregoli, 1997)

[RO] "Eu, îşi spuse micul prinŢ, dacă aş avea de irosit cincizeci si trei de minute, aş porni în linişte spre o fântână." (trad. par Benedict Corlaciu, 1998) ${ }^{8}$

InterCorp, créé par l'Institut du Corpus national tchèque, contient actuellement des textes en 39 langues $^{9}$, y compris des langues romanes (le français, le portugais, l'espagnol, l'italien, le catalan et le roumain), au total 1597462625 mots. La langue tchèque constitue la langue pivot du corpus (chaque texte est aligné par rapport à la version tchèque), mais dans les recherches sur corpus, il est possible de mettre le tchèque de côté et d'effectuer des recherches directement sur les langues choisies (par exemple sur le français et l'espagnol) ${ }^{10}$. Étant donné le caractère synchronique du corpus, seulement les textes écrits après la Seconde Guerre mondiale sont admis ${ }^{11}$.

Pour présenter les principes méthodologiques de travail sur corpus parallèles, le corpus InterCorp présente plusieurs avantages :

1) lancé en 2008, ce corpus a déjà été mis à l'épreuve par de nombreuses recherches, dont certaines réunies dans des actes de trois colloques internationaux qui y ont été consacrés (cf. par exemple Čermák - Klégr - Corness 2010, et la base de publications disponible sur http://biblio.korpus.cz/);

2) par sa taille et par sa composition, InterCorp respecte dans les limites du possible les critères de la représentativité définis en linguistique de corpus (cf. section 2) ;

3) l'accès au corpus est gratuit (après enregistrement - https://www.korpus.cz/ toolbar/signup.php) ce qui permet à chaque lecteur de cet article de tester dans l'immédiat les principes méthodologiques présentés.

L'accent mis dans cette analyse sur les inconvénients possibles des corpus parallèles ne devrait pas éclipser la grande utilité de ce nouvel outil de la recherche linguistique. Les corpus parallèles ont en effet déjà trouvé une large application dans de nombreux domaines : tout en ayant pour domaine de prédilection la recherche contrastive (cf. cidessous), les corpus parallèles sont très utiles aussi dans la lexicographie bilingue ou dans l'enseignement de langues (Botley - McEnery - Wilson 2000, Kratochvílová 2015, même au niveau universitaire - Nádvorníková et al. 2010). En outre, les bases de données parallèles permettent de développer des outils informatiques dans le domaine du TALN alignement au niveau de phrases/mots, la traduction automatique statistique (statistical machine translation), extraction de lexiques multilingues, projection de l'annotation morphosyntaxique sur d'autres langues, désambiguïsation du sens des mots (word-sense disambiguation), etc.

Dans le présent article, nous nous intéresserons en particulier à deux domaines d'application de corpus parallèles, à savoir la traductologie et la linguistique contrastive. L'utilité des corpus parallèles pour la traductologie a été pointée par Baker (1993) et depuis, plusieurs traductologues ont commencé à s'en servir (Olohan 2004, Laviosa 2006, Ballard 2007, etc.). Cependant, c'est en linguistique contrastive que les corpus parallèles ont connu le plus grand succès. Comme le constatent Altenberg et Granger (2002: 7), les corpus parallèles ont donné un nouvel élan aux recherches contrastives en leur offrant de larges données riches et fiables ${ }^{12}$. En effet, les corpus parallèles permettent de voir le sens à travers la traduction (Johansson $2007: 57$ ).

En linguistique constrastive, les corpus parallèles trouvent leur application au niveau morphologique, syntaxique ainsi que lexical : la préposition with et ses équivalents en allemand (Schmied - Fink 2000), aller - go et leurs collocations (Barlow 2008), le préfixe 
verbal re- en espagnol et ses équivalents en tchèque (Čermák 2013), l'expression du temps en français et en italien (Hédiard 2005), les verbes mentaux en polonais et en tchèque (Kaczmarska et al. 2015), les diminutifs en allemand, en anglais et en tchèque (Káňa 2011), etc $^{13}$. Les corpus multilingues permettent en plus de comparer plusieurs langues en même temps ${ }^{14}$. Cette approche devient particulièrement intéressante s'il s'agit de langues typologiquement différentes : une recherche récente a ainsi été consacrée à l'analyse de cinq phénomènes linguistiques (les gérondifs, les constructions causatives et inchoatives, les préfixes verbaux re-/ri- et les suffixes adjectivaux -ble/-bile) en italien, en français, en espagnol et en portugais, par rapport à leurs équivalents en tchèque (Čermák Nádvorníková et al. 2015).

Pour illustrer les problèmes méthodologiques liés à l'utilisation des corpus parallèles, nous allons nous servir de ces recherches concrètes déjà réalisées sur le corpus parallèle InterCorp. Il s'agira en particulier de l'analyse du gérondif français et de ses équivalents en tchèque (Nádvorníková 2010b et d'autres) et de l'analyse présentant le résultat de la coopération entre la linguistique contrastive et la traductologie (voir Nádvorníková Šotolová 2016, à paraître), consacrée aux changements de la ponctuation dans les traductions du français en tchèque et vice versa.

L'article se subdivise en quatre parties. Dans les deux premières sections, nous allons nous concentrer sur les problèmes méthodologiques liés à la taille et à la composition des corpus et à certaines spécificités techniques de corpus parallèles (qualité de l'alignement, gestion de l'étiquetage morphologique dans plusieurs langues, etc.). Dans les deux sections suivantes, nous allons d'abord présenter les facteurs extratextuels susceptibles d'influencer la langue de la traduction, pour finir par l'analyse des spécificités de la langue de traduction per se.

\section{Problèmes méthodologiques liés à la taille et à la composition de corpus parallèles}

Les critères de la taille et de la composition de corpus sont largement débattus dans l'ensemble de la linguistique de corpus (à partir de Biber 1993), parce qu'ils déterminent la représentativité des corpus en question. Dans le cas de corpus parallèles, cette question est encore plus épineuse, et cela pour deux raisons :

1) la taille des corpus parallèles ne peut jamais atteindre celle des plus larges corpus unilingues, tels que FRANTEXT, BNC ou le Corpus national tchèque (cf. www.korpus.cz), étant donné que leur création nécessite un traitement spécial (en particulier l'alignement) et que les textes qui peuvent y entrer sont limités aux traductions ;

2) limités par définition aux textes traduits, les corpus parallèles couvrent avec difficulté certains registres (surtout la langue parlée) et certains types de textes (les textes journalistiques et scientifiques, par exemple, sont moins souvent traduits que les textes littéraires).

Les créateurs du corpus InterCorp font face à ces contraintes limitant la pertinence des résultats obtenus en essayant d'accroître constamment la taille du corpus et en élargissant l'éventail des types de textes couverts ${ }^{15}$. Ainsi, comme nous l'avons déjà mentionné, le corpus contient actuellement plus d'un milliard et demi de mots (au total pour les 39 langues $)^{16}$, mais il ne cesse de croître, et l'Institut du Corpus national tchèque lance chaque année une nouvelle version élargie et améliorée (voir http:// ucnk.ff.cuni.cz/intercorp/?lang=en). A part les textes littéraires (et scientifiques), 
représentant le noyau du corpus, InterCorp offre aux utilisateurs également des « collections » de textes, issues de plusieurs projets. Les proportions entre les différentes parties du corpus sont présentées dans le tableau suivant :

\begin{tabular}{|l|l|l|l|l|l|}
\hline $\begin{array}{l}\text { Noyau du corpus } \\
\text { (litt./scientif.) }\end{array}$ & $\begin{array}{l}\text { Syndicate/ } \\
\text { Presseurop }\end{array}$ & $\begin{array}{l}\text { Acquis } \\
\text { communautaire }\end{array}$ & Europarl & Subtitles & TOTAL \\
\hline 278773665 & 51177809 & 450498235 & 277951947 & 539060966 & 1597462625 \\
\hline $17 \%$ & $3 \%$ & $28 \%$ & $17 \%$ & $34 \%$ & $100 \%$ \\
\hline
\end{tabular}

TABLEAU 1. Composition du corpus parallèle InterCorp (39 langues) - nombre de mots.

Comme le montre clairement le Tableau 1, les différents sous-corpus sont de taille très variée :

- le sous-corpus le plus large, représentant plus d'un tiers de l'ensemble du corpus, est constitué par la collection Subtitles (www.opensubtitles.org), composée de sous-titres de films ;

- les textes de l'Acquis communautaire (https://ec.europa.eu/jrc/en/institutes/ipsc/ ? id $=198$; Erjavec et al. 2005) constituent le deuxième sous-corpus le plus large ${ }^{17}$;

- EuroParl - transcriptions des débats réalisés au sein du Parlement européen entre les années 2007-2011 (http://www.statmt.org/europarl//) ${ }^{18}$ constituent $17 \%$ de la totalité du corpus ;

- le «noyau» du corpus composé de textes littéraires et scientifiques occupe la même proportion que Europarl (17\%, 278 millions de mots) ; l'accent est mis sur les textes littéraires, les textes scientifiques étant donc assez peu représentés ;

- les moins représentés sont les textes journalistiques, tirés des serveurs multilingues SYNDICATE (http://www.project-syndicate.org/) et VoxEurop (PressEurop, (http:// www.voxeurop.eu/fr) - textes parallèles en allemand, anglais, espagnol, français, italien, néerlandais, polonais, portugais, roumain et tchèque.

En ce qui concerne la section française du corpus, qui nous intéresse davantage dans cette analyse (cf. 4), les proportions des différents sous-corpus sont similaires à celles de l'ensemble du corpus InterCorp :

\begin{tabular}{|l|l|l|l|l|l|}
\hline $\begin{array}{l}\text { Noyau du corpus (litt./ } \\
\text { scientif.) }\end{array}$ & $\begin{array}{l}\text { Syndicate/ } \\
\text { Presseurop }\end{array}$ & $\begin{array}{l}\text { Acquis } \\
\text { communautaire }\end{array}$ & Europarl & Subtitles & TOTAL \\
\hline 9170042 & 7321278 & 27351591 & $\begin{array}{l}17178 \\
444\end{array}$ & $\begin{array}{l}25961 \\
848\end{array}$ & $\begin{array}{l}86983 \\
203\end{array}$ \\
\hline $11 \%$ & $8 \%$ & $31 \%$ & $20 \%$ & $30 \%$ & $100 \%$ \\
\hline
\end{tabular}

TABLEAU 2. Composition du corpus parallèle InterCorp (français) - nombre de mots.

En comparant les Tableaux 1 et 2 , nous pouvons constater de légères différences de proportions des sous-corpus, mais les trois ensembles les plus représentés sont toujours les deux sous-corpus de textes issus des institutions de l'Union européenne (Acquis communautaire et Europarl) et le corpus spécifique de sous-titres de films. Les textes 
littéraires et scientifiques, censés être les plus représentatifs pour la langue donnée, représentent ici seulement $11 \%$ du corpus (plus de 9 millions de mots).

Les différences de taille observées entre les sous-corpus n'empêchent pas d'effectuer des recherches sur le corpus et d'obtenir des résultats très pertinents, mais elles exigent que l'utilisateur respecte deux règles méthodologiques simples, valides aussi pour des recherches sur corpus unilingues :

[1] en présentant les résultats obtenus sur l'ensemble du corpus parallèle InterCorp (pour la langue donnée), il faut toujours prendre en considération (et mentionner explicitement) le fait que la moitié d'entre eux est basée sur les textes issus des institutions de l'Union européenne et qu'un tiers est tiré du sous-corpus spécifique de sous-titres de films ;

[2] en comparant les résultats obtenus sur les différents sous-corpus (du corpus parallèle InterCorp), il faut toujours prendre en considération (et mentionner explicitement) les différences de taille entre eux.

A ces deux règles de base nous pouvons en ajouter une troisième, qui est largement acceptée dans l'ensemble de la linguistique de corpus, mais qui peut s'avérer particulièrement pertinente dans le cas de recherches sur corpus parallèles :

[3] Les résultats non-parallèles obtenus sur des corpus parallèles devraient être si possible vérifiés sur des corpus unilingues (représentatifs) des langues et des types de textes respectifs, pour découvrir d'éventuelles spécificités liées aux idiolectes des auteurs ou des traducteurs ${ }^{19}$.

En effet, l'avantage des corpus parallèles est de proposer des occurrences parallèles équivalents des phénomènes linguistiques analysés, mais étant donné la taille nécessairement limitée des corpus parallèles (voir ci-dessus), ces données sont plus susceptibles d'être influencées par des idiolectes spécifiques des auteurs dont les œuvres sont incluses dans le corpus. La règle [3] concerne donc en particulier les sous-corpus parallèles de textes littéraires et scientifiques, dont la taille est plus restreinte que celle des « collections ", telles que Subtitles ou Acquis communautaire.

Pour illustrer l'utilité de ce principe méthodologique, prenons l'exemple de notre recherche de l'usage de la ponctuation en français et en tchèque (Nádvorníková Šotolová 2016, à paraître), effectuée sur le noyau du corpus parallèle InterCorp (cf. Tableau 2). L'objectif de notre analyse était d'identifier les différences d'emploi des signes de ponctuation en français et en tchèque et d'analyser les modifications de la structuration en phrases opérées par les traducteurs. Nous n'allons pas ici présenter en détail les résultats de notre analyse, mais montrer les problèmes méthodologiques qu'il a fallu résoudre au cours de sa réalisation. Un de ces problèmes était effectivement la taille relativement restreinte du sous-corpus français-tchèque, l'analyse étant limitée au noyau du corpus, qui assure la meilleure qualité de la traduction (contrairement au sous-corpus de Subtitles) et permet toujours d'identifier le sens de la traduction (pour l'importance de ce critère, voir section 4.3.1). Nous avons en plus exclu du noyau du corpus les traductions basées sur un original d'une autre langue que le français ou le tchèque (voir 4.3.1). Le corpus final contenait ainsi 61 textes originaux français (52 textes littéraires 3555295 positions, et 9 textes scientifiques -860146 positions ${ }^{20}$ ) ; le corpus tchèque était composé seulement de 21 textes (1532 725 positions en tchèque/1 734687 positions en français, uniquement des textes littéraires). (Le terme position désigne non seulement les mots-occurrences, mais aussi les signes de ponctuation.)

Pour identifier d'éventuelles spécificités de ces deux sous-corpus, nous avons choisi comme corpus de comparaison pour le tchèque le Corpus national tchèque SYN2010 ( 
www.korpus.cz), concrètement les textes originaux littéraires parus après 1950 (16 074 858 positions). Pour le français, nous avons utilisé le sous-corpus de textes littéraires (romans) et scientifiques (séparément) du corpus FRANTEXT (31610109 positions, www.frantext.fr), également limités par la date de parution (après 1950). Pour illustrer le principe [3], nous allons présenter seulement les résultats obtenus pour les textes littéraires français :

\begin{tabular}{|l|l|l|l|l|l|l|l|}
\hline Corpus & $\begin{array}{l}\text { point } \\
\text { final }\end{array}$ & $\begin{array}{l}\text { point } \\
\text { d'interrogation }\end{array}$ & $\begin{array}{l}\text { point } \\
\text { d'exclamation }\end{array}$ & virgule & $\begin{array}{l}\text { points de } \\
\text { suspension }\end{array}$ & $\begin{array}{l}\text { deux- } \\
\text { points }\end{array}$ & $\begin{array}{l}\text { point- } \\
\text { virgule }\end{array}$ \\
\hline $\begin{array}{l}\text { FRANTEXT } \\
(31610109 \text { positions) } \\
\text { (ipm) }\end{array}$ & 42835 & 4722 & 3941 & 70325 & 4403 & 4126 & 1933 \\
\hline $\begin{array}{l}\text { InterCorp positions) } \\
\text { originaux en fr. } \\
(3555295 \quad 39890 \\
(\text { ipm })\end{array}$ & 5848 & 10720 & 62958 & 14005 & 3858 & 1199 \\
\hline
\end{tabular}

Tableau 3. Fréquence relative (ipm - item per million) des signes de punctuation dans la partie française du noyau du corpus parallèle InterCorp (originaux français) et dans le sous-corpus de romans parus après 1950 du corpus FRANTEXT.

Le Tableau 3 montre que la taille du corpus de comparaison (en l'occurrence FRANTEXT) est dix fois plus grande que celle du sous-corpus des originaux français contenus dans le corpus parallèle InterCorp. Les colonnes du Tableau 3 indiquent les fréquences relatives (ipm - items per million) des différents signes de ponctuation dans les sous-corpus analysés, les encadrés indiquant les différences les plus importantes sur lesquelles nous allons nous pencher en détail.

Les différences les plus marquées entre le corpus parallèle InterCorp et le corpus FRANTEXT sont observables dans le cas de deux signes de ponctuation: le point de suspension et le point d'interrogation. En regardant la composition du sous-corpus InterCorp de plus près, nous en découvrons tout de suite la cause: deux romans volumineux de Louis-Ferdinand Céline (Nord et D'un château à l'autre), dans lesquels la fréquence relative de ces deux signes de ponctuation dépasse plus de deux/trois fois la moyenne observée dans le sous-corpus de romans de FRANTEXT :

(1) je peux pas tendre la main !... même pour les A.S... même les A.M.C... je démordrai pas !... idiot d'orgueil! (Louis-Ferdinand Céline, D'un château à l'autre, 2000/1957)

> nedokážu natáhnout ruku !... ani na pokladnu... ani na bezplatnou lékařskou péči... budu si paličatě stát na svým !... pitomá pýcha! (trad. par Anna Kareninová, 1996)

Le style spécifique du roman, basé sur des exclamations du narrateur, explique la fréquence élevée des points d'exclamation et des points de suspension. Leur fréquence absolue très élevée influence ensuite l'ensemble du corpus ${ }^{21}$. Ces spécificités du corpus parallèle ne nous empêchent pas du tout d'y effectuer des recherches contrastives (et traductologiques) pertinentes, elles ne font que démontrer que les données quantitatives non-parallèles extraites des corpus parallèles peuvent différer des usages observés sur des corpus plus larges et qu'il faut respecter la règle [3] indiquée ci-dessus. 


\section{Facteurs techniques de la méthodologie du traitement et de l'exploitation de corpus parallèles}

Dans la section 2, nous avons présenté les problèmes méthodologiques que les corpus parallèles partagent avec toute recherche sur corpus: même les recherches sur corpus unilingues doivent prendre en considération la taille et la composition du corpus sur lequel elles sont basées et tenir compte de l'influence possible des particularités des ouvrages dont le corpus est composé. Les sections 3 et 4 par contre seront consacrées aux problèmes spécifiques des corpus parallèles, dûs à la présence de textes traduits - tant au niveau technique (section 3) que linguistique et traductologique (section 4).

Parmi les facteurs techniques de l'exploitation de corpus parallèles ${ }^{22}$, le plus important est celui de la qualité de l'alignement des versions parallèles. Dans le corpus InterCorp, comme dans la majorité des corpus parallèles, l'alignement est opéré au niveau de phrases : la première tâche consiste donc à identifier leurs frontières ce qui parfois n'est pas évident, comme le montre par exemple (1). En outre, les outils informatiques (les segmentateurs) utilisés pour les différentes langues ne définissent pas la frontière de la phrase (du segment) de la même façon; ainsi, le segmentateur Tokenize, analysant le tchèque, considère le point-virgule comme signe ouvrant un nouveau segment, contrairement à Punkt (Punkt sentence tokenizer, cf. Kiss - Strunk 2006) pour le français ${ }^{23}$.

L'alignement automatique des textes pour le corpus InterCorp se fait par le logiciel hunalign (cf. Varga et al., 2005, http://mokk.bme.hu/resources/hunglishcorpus/), qui donne des résultats assez fiables. Dans les textes du noyau du corpus, la qualité de l'alignement est encore améliorée par le contrôle semi-manuel dans l'éditeur de textes parallèles nommé InterText (cf. Vondřička 2014, http://wanthalf.saga.cz/intertext). Ainsi, dans un ensemble de 4000 non $1: 1^{24}$ segments du noyau du corpus InterCorp, seulement $2,5 \%$ étaient mal alignés. Cependant, dans les « collections » du corpus InterCorp et dans d'autres corpus parallèles, où le contrôle de l'alignement automatique n'est pas effectué, le pourcentage de segments manquant d'équivalent correct peut être plus élevé.

[4] L'utilisateur de corpus parallèles devrait se renseigner sur la qualité de

l'alignement offerte ${ }^{25}$.

En outre, les différences de traitement ne concernent pas seulement les segmentateurs, mais aussi les étiqueteurs morphosyntaxiques utilisés pour les langues incluses dans le corpus parallèle, parce qu'ils diffèrent tant au niveau de la fiabilité des résultats qu'au niveau des ensembles d'étiquettes (tagsets) utilisés. Dans le corpus InterCorp, nous pouvons ainsi observer des différences entre le logiciel TreeTagger (www.ims.unistuttgart.de/projekte/corplex/TreeTagger), utilisé pour le français, dont les résultats sont parfois discutables, et l'annotateur Morče (http://ufal.mff.cuni.cz/morce), qui a été créé spécifiquement pour le tchèque, langue morphologiquement complexe, et dont le taux de fiabilité est très élevé ${ }^{6}$. En formulant sa requête CQL (Corpus Query Language), l'utilisateur doit en plus gérer la nécessité de maîtriser deux (ou plusieurs) ensembles d'étiquettes morhosyntaxiques, dans lesquels la même dénomination peut cacher des réalités linguistiques très différentes :

(2) CS - Morče :

Nepotřebuješ svoje /P8F-4----------- oči, králi ! (Milan Kundera, Žert, 1991/1967)

FR - TreeTagger : 
Tu n'as pas besoin de tes /DET :POS yeux, mon roi! (La Plaisanterie, trad. par Marcel Aymonin, 1991)

L'exemple (2) montre que le système positionnel tchèque spécifie pour le mot en question (svoje) plusieurs facteurs - la partie du discours et son sous-type, le genre et le cas. L'annotation en français, par contre, précise seulement la partie du discours et son soustype (déterminant possessif). Mais la différence la plus frappante est due aux différences de traditions linguistiques: le tchèque ne disposant pas de déterminants, le mot svoje (déterminant possessif en français) est dans les grammaires tchèques traité dans la classe des pronoms ${ }^{27}$. Cet exemple montre le besoin d'un cinquième principe :

[5] l'utilisateur de corpus parallèles désirant se servir de l'étiquetage morphosyntaxique devrait toujours consulter la description officielle des ensembles d'étiquettes (tagsets) utilisés et prendre en considération les différences de traditions linguistiques des langues analysées.

Les aspects techniques de la méthodologie d'exploitation de corpus parallèles sont relativement faciles à identifier; par contre, les facteurs influençant le processus de la traduction et son résultat, et les spécificités de la langue de traduction en tant que telle, demandent plus de subtilité.

\section{Facteurs traductologiques de la méthodologie de l'exploitation de corpus parallèles}

Pour simplifier cet exposé, nous allons diviser les facteurs traductologiques de l'exploitation de corpus parallèles en deux grands groupes: d'une part, il s'agit des facteurs externes au processus de la traduction et de sa publication (4.2), d'autre part, ce sont des spécificités de la langue de traduction, soit dues aux influences de la langue source sur la langue cible (des « interférences », 4.3.1), soit indépendamment des paires de langues concernées (les «universaux» de la traduction, cf. 4.3.2.). Toutes ces influences risquent soit d'éloigner la langue de traduction de la langue cible, aboutissant à un "troisième code » (Øverås : 1998) ou «translationese » (Schmied - Schäffler 1996), soit d'entraîner des non-équivalences avec le texte source. Avant de nous lancer dans l'analyse de ces facteurs de la traduction, il faut donc au moins brièvement aborder la question même de l'« équivalence » de la traduction avec l'original.

\subsection{La question d'« équivalence » des versions parallèles}

Le sujet d'équivalence des segments parallèles est très complexe et a déjà fait couler beaucoup d'encre (par exemple Guidère 2011), en particulier dans le domaine des recherches traductologiques. En ce qui concerne les recherches sur corpus parallèles, les traductologues font à juste titre remarquer que les analyses s'y limitent trop souvent à la comparaison des expressions séparées, ou bien, dans le meilleur des cas, au contexte d'une seule phrase - mais le traducteur ne traduit pas des expressions séparées ou des phrases, mais un texte $e^{28}$. Par conséquent, les causes de certaines «non-équivalences » des segments comparés peuvent être retrouvées seulement en consultant un contexte plus large (dans le cas extrême, le texte entier). A titre d'exemple, le traducteur peut changer la structure d'une phrase ou modifier/remplacer une expression pour éviter sa répétition ${ }^{29}$; il peut également compenser un changement ailleurs dans le contexte (par exemple, le trait «familier » d'une expression peut être effacé dans une expression s'il est repris par 
une autre expression dans la phrase suivante/précédente : le caractère global du passage est conservé). Donc,

[6] Avant d'énoncer des jugements critiques concernant la qualité du travail des traducteurs, les utilisateurs de corpus parallèles devraient consulter le contexte le plus large possible des éléments analysés, pour voir si les non-équivalences observées ne sont pas explicables par des compensations ou par la volonté d'éviter des répétitions.

Même dans le cas où les phrases parallèles peuvent être considérées comme équivalentes sans recourir au contexte, l'analyse contrastive ou lexicographique des structures contenues peut être difficile, à cause des procédés de traduction spécifiques qui peuvent être appliqués :

(3) A quelle perversion obscure avez-vous cédé en fournissant à l'humanité, de votre plus belle plume, un acte d'autoaccusation d'une transparence aussi criante? (Amélie Nothomb, L'Hygiène de l'assassin, 2001)

> Jaká ničivá zvrácenost vás přiměla sepsat pro lidstvo [Quelle pervesion destructrice vous a fait écrire] nejčistším stylem, jakého jste schopen, tak křiklavě průhledné sebeobvinění? (trad. par Jarmila Fialová, 2001).

L'équivalent tchèque du gérondif français illustre dans ce cas concret le procédé de traduction nommé modulation (Vinay - Darbelnet $1977: 51$ ) : « une variation dans le message, obtenue en changeant de point de vue, d'éclairage ». Dans le cas du gérondif, il s'agit d'un type d'équivalent original, mais isolé. Pour les traductologues, cet exemple peut être intéressant, mais pour les linguistes cherchant à identifier le ou les équivalent(s) du gérondif français dans le système de la langue tchèque, l'exemple (3) ne présente que très peu d'intérêt. En effet, en linguistique contrastive, il faut laisser de côté les équivalents spécifiques et rares, et se concentrer par contre sur les types d'équivalents les plus fréquents : les patrons de traduction récurrents (recurrent translation patterns, Krzeszowski 1990 : 27), capables de révéler des équivalences systémiques ${ }^{30}$. Ainsi, pour le gérondif français, l'équivalent dominant en tchèque est le verbe fini, ce qui correspond aux caractéristiques typologiques du tchèque, qui préfère le verbe fini aux constructions nominales (le transgressif ou un SN/SP). Sur un ensemble de 2362 gérondifs français dans le sous-corpus français du corpus InterCorp (voir Čermák - Nádvorníková 2015 et al. : 209210), $42 \%$ avaient pour équivalent en tchèque le verbe fini en proposition coordonnée (cf. 4a), $25 \%$ le verbe fini en proposition subordonnée, $14 \%$ des SN ou des SP (cf. $4 \mathrm{~b}$ ) et seulement $2 \%$ un transgressif (4c, cf. 4.3.1) (31 $^{31}$

(4) ... dit-il en souriant.

(4a) > řekl a usmál se. [il a dit et il a souri] (proposition coordonnée, $67 \%$ )

(4b) > řekl s úsměvem. [dit-il avec sourire] (SP, $10 \%)$

(4c) > řekl usmívaje se. (transgressif, vieilli, $2 \%$ )

Sur l'ensemble des gérondifs analysés, seulement dans $5 \%$ des cas les procédés de traduction spécifiques, tels que dans (3) et d'autres choix des traducteurs ont empêché d'identifier un équivalent concret en tchèque (Čermák - Nádvorníková 2015 et al. : 209). La proportion comparable a été observée pour d'autres phénomènes linguistiques et dans d'autres paires de langues (op.cit.), nous pouvons donc conclure que la majorité des segments parallèles sont pertinents pour la recherche contrastive. Ainsi,

[7] dans toute recherche contrastive sur corpus parallèle, il faut distinguer les équivalents censés refléter les équivalences systémiques (recurrent translation equivalents) des types d'équivalents isolés, issus de l'application de procédés de traduction spécifiques. 
La concentration sur les équivalents considérés comme systémiques mène certains linguistes travaillant sur corpus parallèles à sous-estimer l'importance du rôle joué par les traducteurs et par tout le contexte de la création de la traduction. Comme nous allons le montrer dans la section suivante, cette attitude risque de fausser considérablement les résultats obtenus.

\subsection{Facteurs extratextuels de la traduction}

Dans son livre intitulé Introducing Corpora in Translation Studies, M. Olohan prône pour la prise en compte du rôle important des traducteurs et dénonce le fait que les études contrastives mentionnent souvent seulement le nom de l'auteur du texte original, négligeant celui du traducteur (Olohan 2004 : 28). Dans la section 2, nous avons montré la nécessité de prendre en considération les idiolectes des auteurs des textes dont le corpus parallèle est composé (voir l'exemple (1)) ; cependant, dans les corpus parallèles, il faut tenir compte en plus des "auteurs» des traductions, étant donné qu'ils peuvent également mettre en œuvre des stratégies spécifiques. Pour illustrer ce phénomène, prenons un exemple concret - celui de la fréquence (relative et absolue) des points de suspension dans le sous-corpus des traductions du français en tchèque :

\begin{tabular}{|l|l|l|}
\hline Points de suspension & $\begin{array}{l}\text { originaux tchèques } \\
(1532725 \text { positions })\end{array}$ & $\begin{array}{l}\text { traductions en français } \\
(1734687 \text { positions })\end{array}$ \\
\hline fq abs & 3190 & $\mathbf{4 2 8 0}$ \\
\hline fq rel (ipm) & 2081 & 2467 \\
\hline
\end{tabular}

Tableau 4. Fréquence des points de suspension dans le sous-corpus de traductions du tchèque vers le français (corpus parallèle InterCorp).

Le Tableau 4 montre que la fréquence des points de suspension est plus élevée dans les traductions françaises que dans les originaux tchèques. En appliquant la règle [3], nous pouvons constater que la fréquence relative dans les traductions françaises est largement inférieure à la fréquence relative observée dans le corpus de comparaison (FRANTEXT romans, cf. Tableau 3, $4403 \mathrm{ipm}$ ). Dans le cas des textes tchèques, nous observons la même différence: dans les textes littéraires du corpus de comparaison SYN2010 (16 millions de positions), la fréquence relative des points de suspension est $4090 \mathrm{ipm}$, donc deux fois plus élevée que dans ce sous-corpus de InterCorp. Il serait donc possible de conclure que dans le corpus analysé (en français ainsi qu'en tchèque), les points de suspension sont moins fréquents que dans les textes littéraires des langues respectives et que les traducteurs français ont tendance à en ajouter (le nombre des points de suspension dans les traductions françaises étant de $25 \%$ plus élevé que dans les originaux tchèques) ${ }^{32}$. Or, en regardant de plus près la répartition de ces changements entre les différentes œuvres contenues dans le corpus, nous découvrirons que cette "tendance » est le résultat du travail de deux traducteurs français seulement (Erika Abrams et Stephan Meldegg) qui, en traduisant ensemble deux pièces de théâtre de Václav Havel (Largo desolato et La fête en plein air), ont systématiquement remplacé tous les tirets originaux (très fréquents dans les deux pièces) par les points de suspension ${ }^{33}$ : 
(5) Já bych vyrobil tatarskou omáčku - načali bychom nějaké dobré víno - má přijít Lucy - tuhle Olda by jistě taky zůstal - cítím, že by mi to udělalo dobře - trochu se odreagovat - přijít na jiné myšlenky - (Václav Havel, Largo desolato, 1990/1984)

> Je ferais de la sauce tartare... On ouvrirait une bonne bouteille... Lucy doit venir... Je suis sûr qu'Olda resterait aussi... J'ai vraiment besoin de me distraire un peu, changer d'idées, parler du bon vieux temps... (trad. par Erika Abrams et Stephan Meldegg, 1985)

Laissons de côté le changement du rythme que cause ce remplacement (le tiret indique plutôt une rupture d'intonation, tandis que les points de suspension plutôt un inachèvement ou une hésitation); les différences observées dans (5) révèlent un autre principe méthodologique général qui devrait être respecté lors de l'exploitation de corpus parallèles, à savoir la prise en compte systématique du rôle du traducteur. Pendant les analyses des occurrences parallèles,

[8] il faut donc systématiquement prendre en compte le facteur «traducteur », pour éviter de tirer des conclusions à partir d'usages spécifiques ${ }^{34}$.

Pour la même raison méthodologique (et par respect pour leur travail), il est souhaitable de mentionner dans les exemples tirés de corpus parallèles non seulement le nom de l'auteur (et le titre de l'ouvrage), mais aussi le nom du traducteur.

Avec le nom du traducteur, on prend en plus en considération tout un éventail d'autres facteurs susceptibles d'influencer la traduction. Certains concernent directement le traducteur: son âge, son sexe, sa langue maternelle ${ }^{35}$, son expérience - s'agit-il d'un traducteur professionnel (comme c'est le cas pour les textes du noyau du corpus InterCorp), ou non-professionnel (comme c'est le cas pour la plupart des Subtitles) ${ }^{36}$ ? D'autres facteurs concernent plutôt le contexte général de la création de la traduction, en particulier les spécificités de la tradition de la traduction dans la littérature nationale de la langue cible. Dans notre analyse des non $1: 1$ segments du corpus français-tchèque et tchèque-français du corpus InterCorp, il semble que les traducteurs français prennent plus de libertés avec le texte original que les traducteurs tchèques ${ }^{37}$. Dans un texte, le nombre de non $1: 1$ segments atteint même presque $50 \%$ :

(6) [1] A v Sarajevu k tomu, to je v Bosně, paní Müllerová.

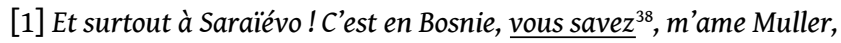

[2] To udělali asi Turci.

[2] et il n'y a que les Turcs qui sont capables de faire un sale coup pareil.

[3] My holt jsme jim tu Bosnu a Hercegovinu neměli brát.

[3] On n'aurait pas dû leur prendre la Bosnie et l'Herzégovine, voilà tout. Ils se vengent à présent.

[4] Tak vida, paní Müllerová. On je tedy pan arcivévoda už na pravdě boží.

[4] Alors notre bon archiduc est monté au ciel, m'ame Muller ? Ça n'a pas traîné, vrai !

(Jaroslav Hašek, Osudy dobrého vojáka Švejka, 1996/1921-1923; trad. par Henry Horejsi, 1932/198939)

La «voix du peuple» présentée par le brave soldat Chvéïk est difficile à traduire; le traducteur a essayé de garder l'oralité de ce discours direct par exemple en ajoutant une expression phatique ([1] vous savez) ou en marquant la prononciation familière de Madame ([1] ou [4]). Cependant, d'autres changements ne trouvent pas leur justification dans l'original et expliquent le nombre élevé de non1:1 segments dans ce texte : d'une part, le traducteur change la segmentation en phrases (cf. par exemple [1] et [2]) ${ }^{40}$, d'autre part, il ajoute des phrases dans le but d'expliciter le sens de l'original (en particulier la deuxième phrase de [3]) ${ }^{41}$. D'autres non1:1 segments apparaissent suite aux omissions (conscientes ou inconscientes) de phrases par les traducteurs - non seulement dans Le Brave soldat Chvéik ${ }^{42}$. 
La traduction du roman célèbre de Jaroslav Hašek nécessiterait une analyse beaucoup plus approfondie que celle que nous pouvons présenter ici; en outre, notre évaluation des changements opérés par le(s) traducteur(s) peut être injuste, parce qu'il faut prendre en considération un autre facteur influençant la traduction, à savoir la date de sa publication (création). En effet, la première traduction française du roman de Jaroslav Hašek a été publiée déjà en 1932. Pour conclure, étant donné que les normes de la traduction et sa position dans la littérature nationale évoluent et que la langue des traductions vieillit plus vite que celle des textes originaux, il est souhaitable de mentionner dans les exemples tirés de corpus parallèles non seulement la date de la parution du texte original, mais aussi celle de la traduction.

Il est évident que l'influence exacte de certains facteurs mentionnés ci-dessus est difficile à cerner, il n'en reste pas moins que

[9] pendant la constitution de corpus parallèles et pendant l'analyse des occurrences parallèles, il est nécessaire de prendre en considération l'éventail le plus large possible de facteurs susceptibles d'influencer la traduction ou d'apporter des informations sur le contexte de sa création et publication ${ }^{43}$.

C'est seulement après avoir pris en compte ces facteurs externes qu'il est possible d'évaluer les spécificités de la langue de traduction en tant que telle - dans une paire de langue donnée ainsi que de manière générale.

\subsection{Spécificités de la langue de traduction}

La langue de traduction peut être spécifique par rapport aux textes originaux de la même langue soit suite à l'influence de la langue source, soit à cause des traits résultant du processus de la médiation linguistique (Baker 1993 : 243, Blum-Kulka 1986 : 21, Johansson 1998: 6), indépendamment de la langue source. Dans le premier cas, il s'agit des « interférences » de la langue source (par exemple des structures syntaxiques françaises calquées sur le tchèque), voir 4.3.1. Le second cas de figure fait l'objet des études des traductologues depuis quelques décennies seulement; dans ce cas, on parle des « universaux de la traduction $»^{44}$. Ces derniers sont capables d'éloigner la traduction non seulement de la langue cible, mais aussi du texte source (voir 4.3.2).

Dans ce qui suit, nous n'allons pas présenter ces deux sortes de spécificités potentielles des textes traduits de manière détaillée; nous allons seulement tenter de proposer des procédés méthodologiques qui pourraient permettre d'éviter qu'elles faussent les résultats obtenus sur corpus parallèles.

\subsubsection{Interférences de la langue source sur la langue cible}

Dans son livre fondateur, G. Toury (1995: 275) définit la «loi d'interférence » comme suit :

« in translation, phenomena pertaining to the make-up of the source text tend to be transferred to the target text "

Toury précise que l'interférence peut apparaître sous forme de transfert négatif (violation des normes de la langue cible) ainsi que positif (augmentation de la fréquence des structures qui existent dans la langue cible), ibid. Nous ajoutons que les interférences fonctionnent tant au niveau lexical que syntaxique, voire orthographique (cf. exemple example entre le français et l'anglais). A titre d'exemple, si les expressions françaises «étoffées" (Vinay - Darbelnet 1977: 109), telles que un chemin menant au village ou 
l'accord conclu entre le Parlement et le Conseil sont traduites en tchèque de manière littérale ( cesta vedoucí do vesnice, smlouva uzavrená mezi Parlamentem a Radou), il s'agit des interférences, parce que le résultat ne correspond pas à l'usage de la langue d'arrivée (en tchèque, il est plus naturel de dire cesta do vesnice, smlouva mezi Parlamentem a Radou ${ }^{45}$.

Pour identifier (et éviter) d'éventuelles interférences, il faut respecter une règle importante de toute recherche sur corpus parallèle, à savoir

[10] toujours prendre en considération le sens de la traduction, c'est-à-dire préciser la langue source et la langue cible ${ }^{46}$, et toujours effectuer les analyses dans les deux sens de la traduction, c'est-à-dire sur des corpus parallèles bi-directionnels ${ }^{47}$.

En effet, si nous travaillons sur des occurrences parallèles unidirectionnelles (par exemple seulement sur des traductions du français vers le tchèque), nous risquons d'en tirer des conclusions influencées par des interférences (ou par des universaux de la traduction, cf. ci-dessous). La situation peut être encore plus compliquée si nous comparons des versions parallèles issues d'une autre langue source, par exemple les traductions française et tchèque d'un texte anglais ${ }^{48}$. Dans ce cas, les versions peuvent être assez éloignées l'une de l'autre, et en plus influencées par les interférences issues d'une troisième langue. Les lexicologues/lexicographes, ayant besoin du nombre d'occurrences extrêmement élevé, se servent souvent de l'ensemble du corpus parallèle, y compris des versions parallèles issues d'une troisième langue ou de «collections » où la langue source ne peut pas être identifiée; néanmoins, dans les recherches contrastives morphosyntaxiques, il est souhaitable de respecter la règle [10].

Pour illustrer l'utilisation du corpus bi-directionnel dans le but de vérifier l'influence potentielle d'interférences, prenons l'exemple de notre recherche déjà évoquée portant sur le gérondif français et ses équivalents en tchèque (cf. 4.1). La recherche contrastive du gérondif est intéressante parce que le présumé équivalent systémique du gérondif en tchèque, le transgressif (forme nominale du verbe - (4c)), est considéré comme vieilli. Pour cette raison, comme nous l'avons déjà mentionné en commentant l'exemple (4), les constructions gérondives sont le plus souvent traduites en tchèque par des constructions à verbe fini, le transgressif représentant seulement $2 \%$ des équivalents. En tchèque contemporain (corpus SYN2010), la fréquence relative du transgressif est $510 \mathrm{ipm}, \mathrm{y}$ compris les formes figées (contre 1640 ipm pour le gérondif, cf. note 21). Dans les traductions du tchèque vers le français, on pourrait donc s'attendre à la fréquence relative du gérondif moins élevée que dans les textes originaux, étant donné que la transposition de la proposition tchèque en construction gérondive française demande au traducteur un effort cognitif plus important que la simple traduction par une proposition à verbe fini. Cependant, en confrontant les fréquences relatives des gérondifs dans les textes originaux français et dans les textes traduits du tchèque, nous observons que les résultats sont comparables (1748 ipm dans les originaux et $1920 \mathrm{ipm}$ dans les traductions). Il semble donc que les traducteurs français soient parfaitement capables d'éviter cette interférence éventuelle du tchèque.

L'analyse bi-directionnelle sur corpus parallèles permet non seulement de vérifier l'influence potentielle des interférences, mais elle représente en plus un des atouts méthodologiques les plus importants de ce type de corpus. En effet, après avoir identifié le type d'équivalent dominant (translation pattern, cf. ci-dessus) dans la langue cible, il est possible de renverser le sens de la traduction et d'utiliser ce type d'équivalent comme «marqueur de sens» (marker of meaning, Malá 2013 et 2014) pour chercher d'autres constructions dans la langue source appartenant au même groupe fonctionnel ${ }^{49}$. Pour 
illustrer ce type d'analyse, prenons comme exemple une recherche réalisée sur le souscorpus anglais-tchèque du corpus InterCorp. Malá (2014) a pris comme point de départ les verbes anglais de type become (become, come, fall, get, go, grow, turn); l'équivalent tchèque dominant (les préfixes inchoatifs de verbes perfectifs, ex. rozzlobit se - se mettre en colère, vynoriit se - émerger, zestárnout - vieillir, etc.) a été ensuite utilisé en tant que marqueur du sens résultatif analysé pour identifier en anglais d'autres structures proches par leur sens de la construction de départ (par exemple les constructions à verbe support telles que take courage, etc.). Sans les données parallèles, ce résultat serait très difficile à obtenir : ainsi, les corpus parallèles nous fournissent les données auxquelles on n'aurait pas accès autrement ${ }^{50}$.

L'analyse bi-directionnelle permet également d'identifier les éventuels universaux de la traduction.

\subsubsection{Universaux de la traduction}

Baker (1993 : 243) définit les universaux de la traduction en tant que traits qui apparaissent typiquement dans les textes traduits plutôt que dans les énoncés originaux et qui ne sont pas issus de l'interférence de systèmes linguistiques spécifiques ${ }^{51}$. Il existe un grand nombre de types d'universaux de la traduction, qui en plus se recoupent parfois, mais parmi les plus cités, nous trouvons les trois suivants : explicitation (cf. le segment [3] dans (6), Blum-Kulka 1986 ou Séguinot 1988 pour les traductions du français en anglais et vice versa), simplification (par exemple Laviosa 1998, Cvrček - Chlumská 2015) ${ }^{52}$, et normalisation (les traits idiosyncratiques de l'original - au niveaux lexical ainsi que syntaxique - sont effacés et rendus conformes aux traits typiques de la langue cible, cf. ci-dessous).

Les universaux de la traduction (par définition) opèrent indépendamment des paires de langues concernées et indépendamment du sens de la traduction; la règle [10] peut donc nous permettre d'éviter de constater des différences entre les langues comparées là où il ne s'agit que d'un trait spécifique à la langue de traduction en général ${ }^{53}$. Nous pouvons illustrer ce principe en revenant à notre analyse de l'usage de la ponctuation en français et en tchèque.

Dans les traductions du français vers le tchèque, nous avons observé des cas où le traducteur tchèque a relié en une seule phrase complexe des phrases simples courtes. Cette intervention dans le style de l'original est particulièrement frappante dans la traduction de L'Étranger d'Albert Camus, où la succession de phrases courtes devrait créer dans la première partie du roman l'impression de la fatalité des événements.

(7) J'ai fait le chemin à pied. J'ai voulu voir maman tout de suite. (Albert Camus, L'Étranger, 1950)

> Došel jsem tam pěšky a hned jsem chtěl vidět maminku. [... à pied et tout de suite j'ai voulu.... (trad. par Miloslav Žilina, 1969)

A partir de ce type d'exemples, nous pourrions conclure que la langue tchèque évite les successions de phrases simples trop courtes. Néanmoins, en observant les traductions dans l'autre sens de la traduction, à savoir du tchèque vers le français, nous avons rencontré des exemples du même phénomène: des unités courtes sont reliées en une phrase «normale» :

(8) Ti soudruzi jezdci. Kde se vzali ? Odkud? (Jáchym Topol, Noční práce, 2001)

> D'où avaient bien pu sortir ces camarades à cheval? (trad. par Marianne

Canavaggio, 2002) 
Le fait que le même phénomène apparait dans les deux sens de la traduction indique qu'il peut s'agir d'un des universaux de la traduction, dans ce cas concret la normalisation - le traducteur tente de rapprocher le texte des traits typiques de la langue cible, mais ce faisant, il éloigne la traduction du style du texte source. En même temps, la langue de traduction peut s'éloigner paradoxalement de la langue cible, étant donné que ses traits typiques sont exagérés. Certains effets de la normalisation, simplification ou explicitation rendant la langue de traduction spécifique par rapport à la langue de textes originaux de la même langue peuvent être découvertes grâce aux corpus comparables de traduction :

[11] avant de nous lancer dans l'analyse contrastive d'un phénomène linguistique sur un corpus parallèle, il est souhaitable de vérifier sur des corpus comparables de traductions si les résultats ne risquent pas d'être faussés par des spécificités de la langue de traduction.

Les corpus comparables de traductions sont composés d'un sous-corpus de textes traduits et d'un autre sous-corpus (comparable quant à la taille et la composition) contenant des textes originaux de la même langue. Pour le tchèque, il existe le corpus Jerome (Chlumská 2013) dans lequel nous pouvons comparer les textes tchèques non-traduits (1 768079 positions) aux textes tchèques traduits à partir de 14 langues (pour les textes littéraires, 1765433 positions) ${ }^{54}$. Dans notre recherche de la ponctuation en français et en tchèque, nous avons ainsi testé la présence d'éventuelles spécificités de la langue de traduction dans l'usage des différents signes de ponctuation:

\begin{tabular}{|c|c|c|c|c|c|c|c|c|}
\hline Jerome & $\begin{array}{l}\text { nombre } \\
\text { de } \\
\text { positions }\end{array}$ & $\begin{array}{l}\text { point } \\
\text { final }\end{array}$ & point d'interrog. & point d'exclam. & virgule & $\begin{array}{l}\text { point de } \\
\text { suspension }\end{array}$ & deux-points & point-virgule \\
\hline $\begin{array}{l}\text { Textes littéraires } \\
\text { orig. fq. abs. }\end{array}$ & 1768079 & 106300 & 11263 & 7044 & 132760 & 4041 & 4977 & 715 \\
\hline $\begin{array}{l}\text { Textes littéraires } \\
\text { orig. fq. rel. }\end{array}$ & & $60122^{55}$ & 6370 & 3984 & 75087 & 2286 & 2815 & 404 \\
\hline $\begin{array}{l}\text { Textes littéraires } \\
\text { trad. fq. abs. }\end{array}$ & 1765433 & 101549 & 11099 & 5741 & 134788 & 6501 & 6400 & 5266 \\
\hline $\begin{array}{l}\text { Textes littéraires } \\
\text { trad. fq. rel. }\end{array}$ & & 57521 & 6287 & 3252 & 76348 & 3682 & 3625 & 2983 \\
\hline chi-carré & & $\begin{array}{l}\text { signif. } \\
\text { à } \quad p \\
<.001\end{array}$ & $\begin{array}{l}\text { difference non } \\
\text { signif. }\end{array}$ & $\begin{array}{l}\text { signif. } \\
\text { à } \mathrm{p}<.001\end{array}$ & $\begin{array}{l}\text { signif. } \\
\text { à } \quad p \\
<.001\end{array}$ & $\begin{array}{l}\text { signif. } \\
\text { à } \mathrm{p}<.001\end{array}$ & $\begin{array}{l}\text { signif. } \\
\text { à } \mathrm{p}<.001\end{array}$ & $\begin{array}{l}\text { signif. } \\
\text { à } \mathrm{p}<.001\end{array}$ \\
\hline $\begin{array}{l}\text { DIN (corpus de } \mathrm{r} \\
\text { textes littéraires o }\end{array}$ & $\begin{array}{l}\text { éférence= } \\
\text { prig.) }\end{array}$ & -2 & -1 & -10 & 1 & 23 & 13 & 76 \\
\hline
\end{tabular}

Tableau 5. Fréquence des signes de ponctuation dans le corpus comparable de traductions tchèques Jerome (cf. Nádvorníková - Šotolová 2016, à paraître). (fq. abs. = fréquence absolue ; fq. rel. = fréquence relative) 
Le Tableau 5 révèle que certains signes de ponctuation sont plus fréquents dans les textes non-traduits tchèques (le point final, les points d'interrogation et d'exclamation), d'autres dans les textes traduits (la virgule, le point de suspension, les deux-points et le point-virgule). Cependant, l'index de différence (DIN) montre que dans la plupart des cas, ces différences ne sont pas importantes, sauf dans le cas du point-virgule, où le DIN dépasse largement le seuil critique de 50. Pendant l'analyse sur corpus parallèle, il faudra donc prêter davantage d'attention aux occurrences des points-virgules pour éviter l'influence des spécificités de la langue de traduction: appliquer l'analyse bidirectionnelle (cf. [10] dans 4.3.1), analyser davantage d'occurrences parallèles pour identifier les causes de la fréquence élevée du point-virgule dans les traductions, comparer les résultats obtenus sur des corpus parallèles aux résultats tirés des corpus unilingues respectifs (cf. [3] dans 2), etc.

Il est théoriquement possible de comparer directement les résultats obtenus sur les larges corpus unilingues des deux langues comparées, mais cette approche présente un danger méthodologique : non seulement il est difficile de trouver des corpus comparables quant à la taille et la composition, mais les résultats peuvent être faussés par les différences typologiques entre les deux langues. A titre d'exemple, dans les textes écrits en tchèque, langue synthétique, le nombre de mots est en moyenne d'un tiers moins élevé que dans les textes correspondants en français, langue analytique (indépendamment du sens de la traduction) - cf. par exemple les deux phrases dans (2). Suite à cette différence, les fréquences relatives obtenues sur les corpus unilingues de ces deux langues sont difficilement comparables : identifiée par rapport au nombre de positions total du corpus, la fréquence relative de l'élément linguistique analysé (par exemple le point final) sera toujours faussée par le fait que les textes écrits en tchèque sont plus «denses » que les textes en français. Sur cet aspect, les corpus parallèles présentent un avantage incontestable, à savoir la possibilité des comparer directement les fréquences absolues, étant donné que les corpus devraient correspondre l'un à l'autre :

\begin{tabular}{|c|c|c|c|c|c|c|c|c|}
\hline $\begin{array}{l}\text { InterCorp } \\
\text { textes } \\
\text { littéraires } \\
\text { ( } 52 \text { textes) } \\
\text { fr>cs }\end{array}$ & $\begin{array}{l}\text { nombre } \\
\text { de } \\
\text { positions }\end{array}$ & $\begin{array}{l}\text { point } \\
\text { d'exclam. }\end{array}$ & virgule & $\begin{array}{l}\text { point } \\
\text { final }\end{array}$ & $\begin{array}{l}\text { points de } \\
\text { suspension }\end{array}$ & $\begin{array}{l}\text { deux- } \\
\text { points }\end{array}$ & $\begin{array}{l}\text { point- } \\
\text { virgule }\end{array}$ & $\begin{array}{l}\text { point } \\
\text { d'interrog. }\end{array}$ \\
\hline $\begin{array}{l}\text { fr }>\text { cs originaux } \\
\text { fq. abs. }\end{array}$ & 3555295 & 38118 & 223872 & 141846 & 49800 & 13717 & 4262 & 20796 \\
\hline $\begin{array}{l}f r>c s \text { originaux } \\
\text { ipm }\end{array}$ & & 10720 & 62958 & 39890 & 14005 & 3858 & 1199 & 5848 \\
\hline $\begin{array}{l}\text { fr>cs } \\
\text { traductions } \\
\text { fq. abs. }\end{array}$ & 3016305 & 36458 & 242658 & 145544 & 49264 & 12920 & 4064 & 20461 \\
\hline $\begin{array}{l}\text { fr>cs traductions } \\
\text { ipm }\end{array}$ & & 12087 & 80449 & 48252 & 16333 & 4283 & 1347 & 6783 \\
\hline chi-carré & & $\begin{array}{l}\text { signif. } \grave{a} \\
p<.001\end{array}$ & $\begin{array}{l}\text { signif. } \\
\grave{a} p \\
<.001\end{array}$ & $\begin{array}{l}\text { signif. ̀̀ } \\
p<.001\end{array}$ & $\begin{array}{l}\text { signif. } \grave{a} \\
p<.001\end{array}$ & $\begin{array}{l}\text { signif. } \\
\grave{a} p \\
<.001\end{array}$ & $\begin{array}{l}\text { signif. } \\
\grave{a} p \\
<.001\end{array}$ & $\begin{array}{l}\text { signif. à } p \\
<.001\end{array}$ \\
\hline
\end{tabular}




\begin{tabular}{|l|l|l|l|l|l|l|l|}
\hline $\begin{array}{l}\text { DIN (corpus de référence }= \\
\text { traductions) }\end{array}$ & -6 & -12 & -9 & -8 & -5 & -6 & -7 \\
\hline
\end{tabular}

Tableau 6. Fréquence des signes de ponctuation dans le corpus parallèle InterCorp - traductions du français vers le tchèque (fr>cs), textes littéraires (cf. Nádvorníková - Šotolová 2016, à paraître).

\begin{tabular}{|c|c|c|c|c|c|c|c|c|}
\hline $\begin{array}{l}\text { InterCorp } \\
\text { textes littéraires } \\
\text { (21 textes) cs>fr }\end{array}$ & $\begin{array}{l}\text { nombre } \\
\text { de } \\
\text { positions }\end{array}$ & $\begin{array}{l}\text { point } \\
\text { d'exclam. }\end{array}$ & virgule & $\begin{array}{l}\text { point } \\
\text { final }\end{array}$ & $\begin{array}{l}\text { points de } \\
\text { suspension }\end{array}$ & $\begin{array}{l}\text { deux- } \\
\text { points }\end{array}$ & $\begin{array}{l}\text { point- } \\
\text { virgule }\end{array}$ & $\begin{array}{l}\text { point } \\
\text { d'interrog. }\end{array}$ \\
\hline $\begin{array}{l}\mathrm{cs}>\mathrm{fr} \text { originaux } \\
\text { fq. abs. }\end{array}$ & 1532725 & 6496 & 132726 & 75795 & 3190 & 9447 & 2029 & 8354 \\
\hline $\begin{array}{l}c s>f r \quad \text { originaux } \\
\text { ipm }\end{array}$ & & 4238 & 86595 & 49451 & 2081 & 6164 & 1324 & 5450 \\
\hline $\begin{array}{l}\mathrm{cs}>\mathrm{fr} \\
\text { traductions fq. } \\
\text { abs. }\end{array}$ & 1734687 & 7079 & 101465 & 72104 & 4280 & 8488 & 3093 & 8686 \\
\hline $\begin{array}{l}\text { cs>fr traductions } \\
\text { ipm }\end{array}$ & & 4081 & 58492 & 41566 & 2467 & 4893 & 1783 & 5007 \\
\hline \multicolumn{2}{|l|}{ chi-carré } & $\begin{array}{l}\text { signif. } \\
\grave{a} p<.05\end{array}$ & $\begin{array}{l}\text { signif. à } \\
p<.001\end{array}$ & $\begin{array}{l}\text { signif. } \\
\text { à } \mathrm{p} \\
<.001\end{array}$ & $\begin{array}{l}\text { signif. à } \mathrm{p} \\
<.001\end{array}$ & $\begin{array}{l}\text { signif. } \\
\text { à } \mathrm{p} \\
<.001\end{array}$ & $\begin{array}{l}\text { signif. } \\
\text { à } \mathrm{p} \\
<.001\end{array}$ & $\begin{array}{l}\text { signif. à } \mathrm{p} \\
<.001\end{array}$ \\
\hline \multicolumn{2}{|c|}{$\begin{array}{l}\text { DIN (corpus de référence est } \\
\text { orig.) }\end{array}$} & 5 & -12 & -2 & 15 & -4 & 22 & 3 \\
\hline
\end{tabular}

Tableau 7. Fréquence des signes de ponctuation dans le corpus parallèle InterCorp - traductions du tchèque vers le français - cs>fr (cf. Nádvorníková - Šotolová 2016, à paraître) ; cf. aussi le Tableau 4 pour les points de suspension.

Les Tableaux 6 et 7 confirment que le nombre de mots du corpus tchèque est toujours plus élevé que celui du corpus français, indépendamment du sens de la traduction. Par conséquent, les fréquences relatives des signes de ponctuation analysés sont presque toujours plus élevés en tchèque qu'en français, même dans le cas où leur fréquence absolue est moins élevée qu'en français (c'est par exemple le cas du point d'exclamation dans les traductions du français vers le tchèque ainsi que du tchèque vers le français). Les deux seules exceptions à cette tendance se trouvent dans les traductions du tchèque vers le français (Tableau 7) : les points de suspension et le point-virgule. La fréquence élevée des points de suspension dans ce sous-corpus a été expliquée dans 4.2 (Tableau 4); nous y avons démontré la nécessité de tenir compte des stratégies spécifiques des traducteurs. Quant au point-virgule, cette différence corrobore les résultats observés dans le Tableau 5 : la fréquence de ce signe de ponctuation en tchèque étant peu élevée, les traducteurs français considèrent comme nécessaire de le réintroduire dans le texte français. Sur ce point, il est intéressant de noter que les fréquences absolues des pointsvirgules dans les traductions du français vers le tchèque et dans leurs originaux sont comparables (Tableau 6) ; les traducteurs tchèques semblent donc respecter la décision de l'auteur français d'utiliser ce signe de ponctuation et ne le suppriment pas massivement, même si les différences d'usage entre les deux langues pourraient justifier ce changement 56 . 


\section{Conclusion}

Les subtilités de l'emploi du point-virgule en tchèque et en français peuvent sembler être un sujet de faible importance, mais elles révèlent l'aspect méthodologique qui est à la base de chaque recherche sur corpus parallèle et que nous avons tenté de formuler dans la règle [7] : à chaque moment, le chercheur doit distinguer les contraintes données par les différences des systèmes des langues comparées et les changements issus de la décision (consciente ou inconsciente) du traducteur. Les différences systémiques font l'objet plutôt de l'analyse contrastive et profitent surtout de larges données quantitatives que les corpus parallèles sont capables de fournir (et des grandes tendances dans les types d'équivalents qu'il est possible d'en dégager); l'analyse des autres différences, ancrée plutôt dans l'approche traductologique, est centrée sur l'analyse détaillée des modifications opérées par le traducteur, dans un contexte large du texte traduit (cf. facteurs externes, 4.2).

Les chercheurs en linguistique contrastive et les traductologues peuvent utiliser les corpus parallèles séparément, chacun retranché dans son domaine, mais il serait profitable pour les deux disciplines qu'ils coopèrent davantage: la linguistique contrastive y gagnerait davantage de subtilité et de précision dans l'évaluation des occurrences parallèles (cf. règle [6], [8] et [9] dans 4.2), et la traductologie pourrait appuyer ses observations sur de larges données quantitatives fournies par les corpus. Ainsi, pour les traductologues, les corpus parallèles cesseraient de servir seulement de "bases à exemples» et pourraient contribuer à une recherche approfondie des spécificités de la langue de traduction (avec le concours des corpus comparables de traductions, cf. 4.3.2, règle [11]), à condition de respecter la règle d'analyse bidirectionnelle (cf. [10]). Enfin, tous les utilisateurs devraient tenir compte des particularités techniques de l'exploitation de corpus parallèles (l'alignement [4] et l'étiquetage bilingue [5]) et des règles de base de toute recherche sur corpus, définies par les critères de représentativité (la taille et la composition des corpus - [1], [2] et [3]).

La complexité de la recherche sur corpus parallèles, suggérée par les principes proposés ci-dessus, peut paraître contraignante, parce que la recherche linguistique contrastive y est inséparable des aspects traductologiques des corpus analysés. Cependant, au lieu d'y voir un inconvénient, nous pouvons le considérer comme un atout, un champ de recherche encore inexploré. Il faut seulement accepter que l'exploration de ce nouveau champ est conditionnée par le respect de certains principes méthodologiques - pour ne pas tomber dans les pièges qui y sont tendus.

\section{BIBLIOGRAPHIE}

Altenberg, B. 1998. "Connectors and sentence openings in English and Swedish », in S. Johansson \& S. Oksefjell (eds), Corpora and cross-linguistic research : theory, method, and case studies. Amsterdam : Rodopi, 115-143. 
Altenberg, B. \& Granger, S. 2002. Lexis in Contrast. Corpus-based approaches. Amsterdam : Benjamins.

Baker, M. 1993. " Corpus Linguistics and Translation studies. Implications and Applications », in M. Baker \& G. Francis \& E. Tognini-Bonelli (eds). Amsterdam/Philadelphia : Benjamins, 233-250.

Baker, M. 1998. « Réexplorer la langue de la traduction : une approche par corpus », Meta : journal des traducteurs / Meta : Translators' Journal 43 (4), 1-10. [online]. URL : http:// www.erudit.org/revue/meta/1998/v43/n4/001951ar.pdf.

Ballard, M. 2007. Les Corpus en linguistique et en traductologie. Arras : Artois Presses Université. Barlow, M. 2008. « Parallel texts and corpus-based contrastive analysis », in M. Á. Gómez Gonzáles \& J. L. Mackenzie \& E. M. González Álvarez (eds). Current trends in contrastive linguistics : functional and cognitive perspectives. Amsterdam : Benjamins, 101-122.

Biber, D. 1993. « Representativeness in corpus design », Literary and Linguistic Computing, 8 (4), 243-257.

Blum-Kulka, S. 1986. « Shifts of Cohesion and Coherence in Translation », in J. House \& S. BlumKulka. Interlingual and Intercultural Communication : discourse and cognition in translation and second language acquisition studies. Tübingen : Narr, 17-35.

Botley, S. P. \& McEnery, T. \& Wilson, A. 2000. Multilingual Corpora in Teaching and Research. Amsterdam : Rodopi.

Čermák, F. \& Klégr, A. \& Corness, P. (eds). 2010. InterCorp : Exploring a Multilingual Corpus. Praha : Nakladatelství Lidové noviny.

Čermák, F. \& Rosen, A. 2012. « The case of InterCorp, a multilingual parallel corpus », International Journal of Corpus Linguistics 13 (3), 411-427.

Čermák, P. 2013. «Las posibilidades del estudio ofrecidas por los corpus paralelos: el caso del prefijo español re-», Romanistica Pragensia XIX. AUC Philologia 2/2013, 123-126.

Čermák, P. - Nádvorníková, O. et al. 2015. Románské jazyky a čeština ve světle paralelních korpusů [Les Langues romanes et le tchèque à la lumière des corpus parallèles]. Praha : Karolinum.

Čermáková, A. \& Fárová, L. 2010. « Keywords in Harry Potter and their Czech and Finnish translation equivalents », in F. Čermák \& A. Klégr \& P. Corness (eds), InterCorp : Exploring a Multilingual Corpus. Praha : Nakladatelství Lidové noviny, 177-188.

Chlumská, L. 2013. Korpus Jerome - comparable corpus of Czech translation and non-translation language. Praha : ÚČNK FF UK, URL : www.korpus.cz.

Chlumská, L. - Richterová, O. 2014. « Překladová čeština v korpusech », Naše řeč 97 (4-5), 259269.

Corness, P. 2010. "Shifts in Czech translations of the reporting verb said in English fiction », in Čermák, Fr. \& Corness, P. \& Klégr, A. (eds), InterCorp : Exploring a Multilingual Corpus. Praha : Nakladatelství Lidové noviny, 159-177.

Cvrček, V. - Chlumská, L. 2015. « Simplification in translated Czech : a new approach to typetoken ratio », Russian Linguistics 39 (3), 309-325.

Erjavec, T. \& Ignat, C. \& Pouliquen, B. \& Steinberger, R. 2005. « Massive Multilingual Corpus Compilation - Acquis Communautaire and Totale ». Paper presented at the $2^{\text {nd }}$ Language \& Technology Conference : Human Language Technologies as a Challenge for Computer Science and Linguistics, Poznań, April 21-23. 
Gast, V. \& Levshina, N. 2014. « Motivating w(h)-clefts in English and German : A hypothesisdriven parallel corpus study ", in Anna-Maria de Cesare (ed.), Frequency, Forms and Functions of Cleft Constructions in Romance and Germanic. Contrastive, Corpus-Based Studies. Berlin : de Gruyter Mouton, 377-414.

Guidère, M. 2011. Introduction à la traductologie : penser la traduction : hier, aujourd'hui, demain. Bruxelles : De Boeck - Duculot.

Halmøy, O. 1982. Le gérondif. Éléments pour une description syntaxique et sémantique. Trondheim : Tapir.

Halmøy, O. 2003. Le gérondif en français. Paris : Ophrys.

Hédiard, M. 2005. « Analyse sur corpus de l'expression du temps en français et en italien : valeur ponctuelle vs valeur durative dans les emplois de « jusqu'à ce que » et de « tant que » ", in G. Williams (ed.), La linguistique de corpus. Rennes : Presses universitaires de Rennes, 169-179.

Hricsina, J. 2015. « Análise corporal do gerúndio no Português atual », Études romanes de Brno, 36 (2), 267-284. URL : https://digilib.phil.muni.cz/bitstream/ handle/11222.digilib/134538/1_EtudesRomanesDeBrno_45-2015-2_19.pdf ?sequence =1.

Johansson, S. 1998. On the role of corpora in cross-linguistic research. In S. Johansson and S. Oksefjell (eds) Corpora and Cross-Linguistic Research, Amsterdam : Rodopi, 3-24.

Johansson, S. 2007. Seeing through multilingual corpora. In : R. Facchinetti (ed.), Corpus Linguistics 25 Years On. Amsterdam - New York : Rodopi.

Johansson, S. \& Hofland, K. 1994. « Towards an English-Norwegian Parallel Corpus », in U. Fries \& G. Tottie \& P. Schneider (eds), Creating and using English lanugage corpora. Amsterdam : Rodopi.

Kaczmarska, E. \& Rosen, A. \& Hana, J. \& Hladká, B. 2015. « A syntactico-semantic analysis of arguments as a method for establishing equivalents of Czech and Polish verbs expressing mental states », Prace Filologiczne, $\mathrm{n}^{\circ}$ LXVII, 135-158.

Káňa, T. 2011. « Deminutiva a deminutivní vyjádření v češtině, němčině a angličtině - hledání hranic » [Deminutives and deminutival expresssions in Czech, German and English - searching for boundaries], in Čermák, F. (éd.). Korpusová lingvistika Praha 2011. Praha : NLN, 168-185.

Kiss, T. \& Strunk, J. 2006. « Unsupervised multilingual sentence boundary detection », Computational Linguistics 32 (4), 485-525.

Kratochvílová, D. 2015. « The use of the parallel corpus InterCorp in foreign language teaching. », in H. Krings \& B. Kühn (eds), Fremdschprachliche Lernprozesse. Erträge des 4. Bremer Symposions zum Fremdsprachenlehren und -lernen an Hochschulen. Bochum : AKS-Verlag Bochum, 278-282.

Kruger, A. 2002. « Corpus-based translation research : its development and implications for general, literary and Bible translation », Acta Theologica Supplementum 2, 70-106.

Krzeszowski, T. P. 1990. Contrasting languages : the scope of contrastive linguistics. Berlin : Mouton de Gruyter.

Laviosa, S. 1998. « Core patterns of lexical use in a comparable corpus of English narrative prose », Meta $43(4), 557-570$.

Laviosa, S. 2006. Corpus-based Translation Studies. Theory, Findings, Applications. Amsterdam/ New York : Rodopi. 
Lewis, D. 2005. « Corpus comparables et analyse contrastive : l'apport d'un corpus français/ anglais de discours politiques à l'analyse des connecteurs adversatifs », in G. Williams (ed.), La linguistique de corpus. Rennes : Presses universitaires de Rennes, 179-193.

Malá, M. 2013. « Translation counterparts as markers of meaning. The case of copular verbs in a parallel English-Czech corpus », Languages in Contrast 13 (2), 170-192.

Malá, M. 2014. « Copular verbs of the become type and the expression of 'resulting' meaning in English and in Czech : A contrastive corpus-supported view ", Acta Universitatis Carolinae Philologica 3. Prague Studies in English 26, 99-115.

Malmkjær, K. \& Windle, K. (eds). 2012. The Oxford Handbook of Translation Studies. Oxford : Oxford University Press.

Marès, A. 1986. « La réception de Hašek/Chvéïk en France », Revue des études slaves 58 (1), 83-91.

Martinková, M. 2014. « Cause and Concern : The Have Construction with the Infinitive Seen through Its Czech Translation Equivalents ", in M. Martinková \& M. Janebová \& J. Macháček (eds). Category and Categorial Changes : The Third Syntactical Plan and Beyond. Olomouc: Univerzita Palackého, 21-46. URL : http://anglistika.upol.cz/fileadmin/kaa/categories2014/ Categories_and_categorial_changes_web.pdf.

McEnery, T. \& Xiao, R. \& Tono, Y. 2006. Corpus-based language studies : an advanced resource book. London : Routledge.

Nádvorníková, O. 2009. «Que font les personnages des romans en parlant? (dit-il en souriant). Typologie des constructions gérondives accompagnant les verbes de dire dans les propositions incises ", in A. Kieliszczyk \& Pilecka, E. (eds.), La perspective interdisciplinaire des études françaises et francophones. Łask : Oficyna wydawnicza LEKSEM, 89-98.

Nádvorníková, O. 2010a. «Les corpus parallèles : L’Espace pour l'analyse contrastive ». Études Romanes de Brno, 31 (1), 7-27.

Nádvorníková, O. 2010b. « The French gérondif and its Czech equivalents », in Fr. Čermák \& P. Corness \& A. Klégr (eds), InterCorp : Exploring a Multilingual Corpus. Praha : Nakladatelství Lidové noviny, 83-96.

Nádvorníková, O. (2013a). « Francouzský gérondif a český přechodník : kontrastivní analýza a jazykové korpusy » [Le gérondif en français et le transgressif en tchèque : l'analyse contrastive et les corpus linguistiques], Jazykovědné aktuality, 50 (3-4), 80-96. URL : http:// jazykovednesdruzeni.cz/JA_2013_3_4.pdf.

Nádvorníková, O. 2013b. «- Paul se rase en chantant, dit-il en bafouillant : Quels types de manière pour le gérondif en français? ", Acta Universitatis Carolinae Philologica 2 (Romanistica Pragensia XIX), 31-44.

Nádvorníková, O. 2013c. « Les gérondifs antéposés : quelles relations avec les contextes de gauche et de droite? » Verbum 35 (1-2), 161-174.

Nádvorníková, O. (à paraître). « Le corpus multilingue InterCorp et les possibilités de son exploitation ", in É. Buchi \& J.-P. Chauveau \& J.-M. Pierrel (eds), Actes du XXVII ${ }^{\mathrm{e}}$ Congrès international de linguistique et de philologie romanes (Nancy, 15-20 juillet 2013), 3 volumes. Strasbourg : Société de linguistique romane/ÉLiPhi.

Nádvorníková, O. \& Polická, A. \& Šotolová, J. \& Vurm, P. 2010. « Využití InterCorpu ve vysokoškolských kurzech francouzské filologie », in Fr. Čermák \& J. Kocek, (eds), Mnohojazyčný korpus InterCorp : Možnosti studia. Praha : NLN, 232-241. 
Nádvorníková, O. \& Šotolová, J. (à paraître). « Změny v segmentaci na věty v překladových textech : analýza dat z francouzsko-českého paralelního korpusu. [Modifications de la segmentation en phrases en traduction : analyse des données du corpus parallèle françaistchèque] », in A. Čermáková \& L. Chlumská \& M. Malá (eds), Jazykové paralely. Praha : ÚČNK/ NLN.

Olohan, M. 2004. Introducing Corpora in Translation Studies. London/New York : Routledge.

Øverås, L. 1998. « In Search of the Third Code : An Investigation of Norms in Literary Translation », Meta : Tranlator's Journal 43 (4), 557-570.

Rosen, A. 2010. « Morphological tags in parallel corpora », in Fr. Čermák \& P. Corness \& A. Klégr (eds), InterCorp : exploring a multilingual corpus. Praha : NLN, 205-234. URL : http:// utkl.ff.cuni.cz/ rosen/public/unitags_pap.pdf.

Rosen, A. 2012. « Mediating between incompatible tagsets », in Ahrenberg et al. (eds), Proceedings of the Workshop on Annotation and Exploitation of Parallel Corpora (AEPC), Volume 10 of NEALT Proceedings Series. Tartu : Northern European Association for Language Technology, 53-62.

Rosen, A. \& Vavř́n, M. 2012. « Building a multilingual parallel corpus for human users », in N. Calzolari et al. (eds), Proceedings of the Eight International Conference on Language Resources and Evaluation (LREC'12). Istanbul : Turkey, 5 2012. European Language Resources Association (ELRA), 2447-2452.

Roukos, S. \& Graff, D. \& Melamed, D. 1995. Hansard French/English. Philadelphia : Linguistic Data Consortium.

Schmied, J. \& Fink, B. 2000. « Corpus-Based Contrastive Lexicography : The Case of English with and its German Translation Equivalents », in S.P. Botley \& T. McEnery \& A. Wilson. Multilingual Corpora in Teaching and Research. Amsterdam : Rodopi, 157-176.

Schmied, J. \& Schäffler, H. 1996. « Approaching translationese through parallel and translation corpora », in C.E. Percy \& C. F. Meyer \& I. Lancashire (eds), Synchronic Corpus Linguistics. Amsterdam and Atlanta : Rodopi, 41-57.

Séguinot, C. 1988. « Pragmatics and the explicitation hypothesis », TTR : Traduction, Terminologie, Rédaction 1 (2), 106-114.

Talmy, L. 2000. Toward a Cognitive Semantics (Vol. II : Typology and Process in Concept Structuring). Cambridge (Mass.) : MIT Press.

Toury, G. 1995. Descriptive translation studies - and beyond. Amsterdam : John Benjamins Publishing Company.

Vanderauwera, R. 1985. Dutch Novels Translated into English : The Transformation of a "Minority" Literature. Amsterdam : Rodopi.

Varga, D. et al. 2005. « Parallel corpora for medium density languages », in Proceedings of RANLP 2005. Borovets : Bulgaria, 590-596. URL : http://www.ldc.upenn.edu/Catalog/docs/LDC2008T01/ ranlp05.pdf.

Vavřín, M. \& Rosen, A. 2008. «InterCorp : A Multilingual Parallel Corpus Project », in Proceedings of the International Conference Corpus Linguistics - 2008, St. Petersburg : St. Petersburg State University, 97-104. URL : http://utkl.ff.cuni.cz/ rosen/public/2008_intercorp_peterburg.pdf.

Véronis, J. (éd.) 2000. Parallel text processing : alignment and use of translation corpora.

Dordrecht : Kluwer. 
Vinay, J.-P. \& Darbelnet, J. 1977. Stylistique comparée du français et de l'anglais. Paris : Marcel Didier.

Vondřička, P. 2014. « Aligning parallel texts with InterText », in N. Calzolari et al. (eds), Proceedings of the Ninth International Conference on Language Resources and Evaluation (LREC'14), European Language Resources Association (ELRA), 1875-1879.

Zanettin, F. 2013. « Corpus Methods for Descriptive Translation Studies », in Procedia - Social and Behavioral Sciences 98, 20-32.

\section{NOTES}

1. Cet article a été réalisé grâce au soutien financier de l'Institut du Corpus national tchèque (projet LM2011023) subventionné par le Ministère de l'Éducation nationale tchèque dans le cadre du projet de Grandes Infrastructures de Recherche, Développement et Innovation.

2. Il faut distinguer les corpus parallèles, composés de textes originaux et de leurs traductions respectives, des corpus comparables, impliquant également deux langues, mais à travers des textes non-traduits (cf. McEnery - Xiao - Tono 2006: 47). Les corpus comparables doivent respecter des mêmes critères de genre, registre, public visé, époque de composition, etc. (par exemple des discours politiques en deux langues d'une époque donnée, cf. Lewis 2005). Le corpus incluant plusieurs langues s'appelle multilingue, qu'il soit parallèle ou comparable (Altenberg Granger 2002 : 8). Il existe également des corpus comparables de traductions (cf. ci-dessous 4.3.2).

3. Le corpus Parasol contient 31 langues (à part les langues slaves, il compte aussi le français, www.slavist.de).

4. Pour d'autres sources, voir par exemple http://aclweb.org/aclwiki/index.php? title=Multilingual_Corpora ou le catalogue de l'ELRA (European Language Resources Association).

5. Le corpus multilingue OPUS contient plus d'une centaine de langues (http://opus.lingfil.uu.se), le corpus InterCorp 39 langues (cf. ci-dessous).

6. Le plus souvent, il s'agit d'une annotation morphosyntaxique (cf. section 3), mais certains corpus sont aussi par exemple annotés syntaxiquement (cf. CzEng - Czech-English Parallel Corpus, http://ufal.mff.cuni.cz/czeng ou PCEDT, Prague Czech-English Dependency Treebank, http:// ufal.mff.cuni.cz/prague-czech-english-dependency-treebank).

7. Cf. en anglais Vavř́n - Rosen 2008, Rosen - Vavřín 2012, Čermák - Rosen 2012 ; en français Nádvorníková 2010a et 2016, à paraître.

8. Le Petit prince est représenté dans le corpus en 25 versions au total : cs be bg de ds el en es fr hi $\mathrm{hr}$ hs hu it la mk nl pl ro ru sk sl sv sy uk; voir https://trnka.ff.cuni.cz/ucnk/intercorp/files/ IntercorpTop200/).

9. ar, be, bg, ca, da, de, el, en, es, et, fi, fr, he, hi, hr, hu, is, it, ja, lt, lv, mk, ms, mt, nl, no, pl, pt, ro, ru, sk, sl, sq, sr, sv, tr, uk, vi, cs (pour voir la taille des différents sous-corpus de ces langues, veuillez consulter http://ucnk.ff.cuni.cz/intercorp/?req=page:info).

10. Pour de plus amples informations sur le traitement et les possibilités d'exploitation du corpus InterCorp, voir Nádvorníková 2016, à paraître.

11. Pour certains textes appartenant au patrimoine universel, des exceptions de la limite de la synchronie ont été autorisées; c'est ainsi que nous pouvons trouver dans le corpus InterCorp Voyage au bout de la nuit de Louis-Ferdinand Céline, Cours de linguistique générale de Ferdinand de Saussure ou Le Brave soldat Chvéik de Jaroslav Hašek.

12. D'après Barlow, les recherches effectuées sur corpus parallèles s'ancrent dans le large courant de recherches grammaticales basées sur l'usage (usage-based approaches to grammar, Barlow 2008 : 203). 
13. Certaines recherches sont davantage centrées sur les textes littéraires, par exemple l'analyse des mots clés dans les romans de J.K. Rowling portant sur Harry Potter et leurs équivalents en finnois et en tchèque (Čermáková - Fárová 2010) ou la variation des verbes de parole dans les propositions incises en anglais et en tchèque (Corness 2010).

14. Cependant, il est également possible d'utiliser le corpus parallèle pour des analyses noncontrastives (cf. Hricsina 2015).

15. Récemment, les textes du corpus ASPAC (The Amsterdam Slavic Parallel Corpus) ont été intégrés dans le corpus InterCorp et rendus disponibles pour des recherches, et l'Institut du Corpus national tchèque envisage d'ajouter à ce corpus également le texte de la Bible et ses traductions.

16. Toutes les langues ne sont naturellement pas représentées par le même nombre de mots (cf. https://wiki.korpus.cz/doku.php/cnk :intercorp :verze8). Parmi les langues les mieux représentées, nous trouvons l'espagnol: ce sous-corpus contient plus de 100 millions de mots dans sa totalité, dont 17 millions dans le noyau littéraire.

17. Dans les sous-corpus de l'Acquis communautaire et Europarl, il n'est pas possible d'identifier la langue source ; cependant, Gast - Levshina (2014 : 379), effectuant des analyses sur Europarl, proposent de considérer les phrases de ce corpus parallèle comme «sémantiquement et pragmatiquement proches d'équivalence ", étant donné la qualité élevée des traductions. Nous soutenons cette hypothèse et proposons de l'appliquer aussi sur le corpus de l'Acquis communautaire.

18. Les collections de Subtitles, Acquis communautaire et Europarl (parmi d'autres) sont disponibles également dans le corpus parallèle en ligne OPUS (cf. note 5), mais l'interface permettant d'effectuer des recherches sur les corpus et de traiter les résultats obtenus offre considérablement moins de fonctionnalités que l'interface KonText du corpus InterCorp, basée sur le logiciel NoSketchEngine.

19. Cf. la recommandation similaire chez Altenberg - Granger (2002:9): «The results based on translation corpora [...] have to be verified on the basis of original text corpora. "

20. Parmi les textes scientifiques dans le sous-corpus français du corpus InterCorp, nous trouvons par exemple Cours de linguistique générale de F. de Saussure, Raisons pratiques, sur la théorie de l'action de P. Bourdieu, ou Dames du XII siècle de G. Duby.

21. Les idiolectes doivent être pris en compte même dans le cas où la fréquence relative (fq. rel.) de l'élément linguistique étudié ne diffère pas considérablement de celle observée sur le corpus de comparaison : à titre d'exemple, la fq. rel. du gérondif dans le corpus InterCorp est $1547 \mathrm{ipm}$ ce qui est comparable au corpus de romans parus après 1950 disponibles dans FRANTEXT (1 640 ipm). Cependant, le chiffre obtenu sur InterCorp cache des cas extrêmes : d'un côté, Le Testament français d'Andreï Makine, où la fq. rel. du gérondif est trois fois plus élevée que la moyenne, de l'autre côté par exemple le roman D'un château à l'autre de L.-F. Céline (cf. ci-dessus (1)), où elle ne représente qu'un cinquième de la moyenne (cf. Čermák - Nádvorníková 2015 et al. : 199-200). Le chercheur doit veiller à ne pas analyser les occurrences venant du même auteur; dans ce cas concret, ces précautions sont d'autant plus nécessaires que l'idiolecte de Makine peut être influencé par la langue maternelle de l'auteur (le russe).

22. Pour les aspects techniques de la création et de l'exploitation de corpus parallèles, cf. aussi Véronis (éd.) 2000.

23. La qualité de l'alignement peut être influencée aussi par la qualité de la correction typographique des textes : un simple remplacement d'une virgule par un point final change la segmentation du passage. Par ailleurs, la non-équivalence des segments alignés peut être due au fait que le traducteur a utilisé pour la traduction une autre édition de l'original que celle qui a été incluse dans le corpus. Néanmoins, pendant le choix de textes pour le corpus, les créateurs du corpus InterCorp tâchent toujours de retrouver l'édition utilisée par le traducteur, les segments 
non-correspondants (non1:1 segments, cf. ci-dessous) sont donc en général le résultat des changements (ou omissions) opérés par les traducteurs - consciemment ou inconsciemment.

24. Les non 1 :1 segments sont les segments parallèles dans lesquels le nombre de segments dans une langue n'est pas le même que celui dans l'autre langue, voir (7) et (8) ; il s'agit le plus souvent des cas où le traducteur a soit relié les phrases originales dans une seule phrase complexe, soit divisé une phrase dans deux ou plusieurs unités phrastiques (cf. 4.3.2). Les non1:1 segments incluent aussi les cas de segments manquants - suite aux omissions ou ajouts des traducteurs (cf. par exemple (6)).

25. Avant de conclure hâtivement par exemple que le traducteur a omis de traduire le segment qui dans le corpus manque d'équivalent.

26. On envisage d'améliorer l'annotation morphosyntaxique des textes français $d u$ corpus InterCorp et d'autres corpus français créés par l'Institut du Corpus national tchèque en utilisant par exemple l'étiqueteur Melt, développé par Benoît Sagot (http://ressources.labex-efl.org/melt ) .

27. De même, la définition des entités considérées comme « interjections » varie d'un système à l'autre. Les créateurs du corpus parallèle InterCorp envisagent d'harmoniser les tagsets utilisés, mais cette tâche est très difficile (à ce sujet, voir par exemple Rosen : 2010 et 2012).

28. De même, l'équivalence peut être évaluée au niveau du mot, de la phrase ou du texte.

29. A titre d'exemple, le pronom relatif který en tchèque est moins fréquent que son équivalent français qui/que, parce que le cumul de plusieurs propositions relatives dans une seule phrase complexe (ou dans un même paragraphe) est en tchèque considéré comme une maladresse stylistique. Par conséquent, le traducteur est parfois obligé de changer la structure de la phrase et supprimer qui/který, pour éviter ces répétitions.

30. Néanmoins, même les types d'équivalents peu fréquents peuvent parfois révéler des «patrons» spécifiques, par exemple les différences dans l'expression de la manière du mouvement: le tchèque, appartenant comme l'anglais aux «satellite-framed languages " (cf. Talmy 2000), exprime la manière du mouvement (i) par le radical du verbe et la direction (j) par un «satellite » - le préfixe ( rrí $_{j} b e ̌ h l_{i}$ ), le français, par contre, faisant partie du groupe des «verbframed languages ", exprime la manière du mouvement par un satellite - le gérondif (il est arrivé en courant $t_{i}$.t L'expression de la manière du mouvement étant peu fréquente, ce type d'équivalent ne représente que $1 \%$ de toutes les occurrences analysées (Čermák - Nádvorníková 2015 et al. : 218), mais il reflète une différence typologique importante.

31. La répartition des types d'équivalents en tchèque confirme la distinction de deux types principaux de gérondifs proposée par Halmøy $(1982,2003)$ : « le type A », basé sur « l'antériorité logique ", qui a pour équivalent en tchèque le plus souvent des propositions subordonnées circonstancielles (de temps, de moyen, de condition, etc.) et «le type B », exprimant une pure circonstance concomitante et ayant pour équivalent en tchèque le plus souvent la proposition coordonnée (ou le SP introduit par la préposition s/avec, cf. (4b)). Cette analyse du gérondif confirme ainsi la constatation de Johansson citée dans la section 1 , à savoir que les équivalents dans la langue cible permettent de révéler les différences de sens cachées par la forme unique dans la langue source. Le même principe méthodologique a été appliqué par exemple dans Martinková 2014. (Pour l'analyse du fonctionnement sémantique du gérondif à travers ses équivalents en tchèque, cf. aussi Nádvorníková $2013 \mathrm{~b}$ et 2013c).

32. Pour la possibilité de comparaison des fréquences relatives entres les sous-corpus tchèque et français, cf. 4.3.2.

33. Dans les deux pièces de théâtre, Havel n'a utilisé aucun point de suspension ; les traducteurs en ont ajouté au total 965.

34. De la même façon qu'on évite l'influence de l'idiolecte d'un seul locuteur dans les corpus unilingues en essayant de travailler sur un corpus d'occurrences provenant de locuteurs les plus variés possible. 
35. Certains traducteurs du tchèque vers le français sont tchèques, par exemple Jan Rubeš, qui a traduit plusieurs textes de Václav Havel.

36. Il ne faut pas oublier que d'autres personnes que le traducteur peuvent apporter des changements au texte traduit : certains auteurs maitrisant la langue cible en question relisent les traductions de leurs textes et demandent aux traducteurs des modifications (c'est surtout le cas de Milan Kundera, mais aussi celui de Patrik Ouředník). Enfin, l'importance des modifications apportées par les correcteurs dans les maisons d'édition reste encore à identifier, mais elle peut être considérable (cf. par exemple Séguinot 1988).

37. Vanderauwera (1985) démontre que l'attitude des traducteurs en ce sens peut varier aussi en fonction du « rapport de force » entre les littératures nationales des langues en question.

38. Les mots/phrases dans la traduction qui n'ont pas d'équivalent direct dans l'original sont soulignés.

39. Pour de plus amples informations sur la réception du roman de Hašek en France, voir par exemple Marès 1986 et tout le numéro thématique de la Revue des Études slaves.

40. Dans la première phrase de [1], il s'agit en plus du changement de la modalité (de la modalité déclarative en modalité exclamative), de même dans [4].

41. La deuxième phrase de [4] est l'explicitation de l'adverbe už (déjà) dans la phrase tchèque ; l'ajout dans le segment [2], par contre, change le sens de l'original, basé sur l'adverbe épistémique asi (peut-être).

42. Dans la traduction du roman de J. Hašek, les omissions sont assez fréquentes, et la qualité générale de la traduction est discutable; le chercheur doit le prendre en considération. Une nouvelle (et meilleure) traduction des Aventures du brave soldat Chvéik, créée par B. Meunier, devrait paraitre en 2016 dans la maison d'édition Gallimard.

43. Dans le corpus InterCorp, les métadonnées affichées pour chaque occurrence sont : les noms de l'auteur et $\mathrm{du} / \mathrm{des}$ traducteur/s, les titres de l'original et de la traduction, pour les deux versions les maisons d'édition et l'année de la publication (en cas de décalage important entre la première date de parution et l'édition utilisée pour le corpus, on précise aussi cette première date), lieu de la publication, variante de la langue en question (par exemple le français de France ou de Martinique, etc.). Dans les « collections » les métadonnées peuvent être réduites.

44. Le terme « universaux » de la traduction est parfois contesté (cf. Kruger 2002 : 99, Malmkjær Windle 2012 : 6 ou Zanettin $2013: 25$ ), mais cette notion reste utile, bien qu'on parle davantage de «normes » ou « tendances ».

45. L'interférence peut influencer par exemple la fréquence des pronoms relatifs en tchèque (cf. note 29) ou celle des déterminants (" pronoms») possessifs (cf. (2)), dont l'usage en tchèque est plus restreint qu'en français. A titre d'exemple, la nuance d'habitude exprimée par le déterminant possessif dans je vais acheter mon journal est en tchèque rendue par le pronom réfléchi si (koupím si noviny), la traduction littérale étant inacceptable (*koupím svoje noviny). Chlumská - Richterová (2014) ont démontré sur le corpus comparable de traductions (Jerome, cf. ci-dessous) que la fréquence des déterminants ("pronoms») possessifs est plus élevée en tchèque dans les textes traduits (issus de plusieurs langues sources) que dans les textes originaux.

46. Dans les exemples parallèles, il est souhaitable de mettre à la première place l'exemple du texte source et à la deuxième place la traduction.

47. Le risque d'interférences peut être diminué aussi en limitant le corpus aux traductions faites par des traducteurs professionnels (donc en évitant Subtitles) et en travaillant sur le corpus contenant les traductions faites par un grand nombre de traducteurs différents (pour " dilluer » leurs idiolectes, cf. ci-dessus (5)).

48. Dans le noyau littéraire du corpus InterCorp, un roman de J.K. Rowling est le texte représenté dans le plus grand nombre de versions parallèles ( 26 : cs be bg ca da de el en es fi fr hr hu it mk nl no $\mathrm{pl}$ pt ro ru sk sl sv sy uk); cependant, à la deuxième place, nous trouvons un texte français - Le 
Petit Prince de Antoine de Saint-Exupéry ( 25 : cs be bg de ds el en es fr hi hr hs hu it la mk nl pl ro ru sk sl sv sy uk). Le premier texte tchèque parmi les plus «cotés » se trouve à la $5^{\mathrm{e}}$ place (Milan Kundera - L'Insoutenable légèreté de l'être, 21 versions parallèles : cs ca da de en es fi fr hr hu it lt mk nl no pl ru sl sv sy uk).

49. Altenberg - Granger (2002: 8) proposent la même méthode bi-directionnelle de l'utilisation de corpus parallèles : By reversing this process, i. e. starting from the range of variants discovered in Language $B$ and observing how these are rendered in language $A$, it is possible to discover paradigms of cross-linguistic correspondences (alternative ways of rendering a particular meaning or function in the target language).

50. L'analyse bi-directionnelle est nécessaire pour toute recherche contrastive: à titre d'exemple, bien que le transgressif ne soit pas l'équivalent dominant du gérondif français, il est comme lui la forme adverbiale du verbe, censée assumer les fonctions comparables - le gérondif pourrait donc être son équivalent dominant en français. Cependant, en renversant le sens de la traduction, nous trouvons que l'équivalent le plus fréquent du transgressif n'est pas le gérondif (17\% des 253 occurrences), mais le participe présent dans sa fonction d'épithète détachée aux nuances circonstancielles (35\%).

51. "features which typically occur in translated text rather than original utterances and which are not the result of interference from specific linguistic systems ".

52. Laviosa (1998) a démontré sur un corpus comparable de traductions (ECC, cf. note 54) que dans les textes journalistiques en anglais, le type/token ratio est plus élevé dans les textes traduits que dans les textes originaux; la simplification au niveau lexical a été observée également dans les traductions tchèques (cf. Crrček - Chlumská 2015). Au niveau syntaxique, nous avons observé la tendance à diviser des phrases longues et complexes en unités plus « digeste » (voir Nádvorníková - Šotolová 2016, à paraître).

53. Pour illustrer l'importance de la prise en compte du sens de la traduction dans les recherches sur corpus parallèles, Olohan (2004 : 26-28) présente l'analyse réalisée par B. Altenberg (1998) qui constate que dans les traductions du suédois en anglais, les adverbiaux sont mis en antéposition. Altenberg attribue cette tendance aux exigeances des systèmes linguistiques des deux langues, mais Olohan ajoute que la même tendance apparaît aussi dans les traductions de l'anglais vers le suédois, ce qui prouve qu'il s'agit d'un trait spécifique de la langue de traduction.

54. Pour l'anglais, il existe par exemple The English Comparable Corpus (ECC), élaboré par S. Laviosa.

55. Les fréquences relatives sont marquées en italique, la plus élevée est en plus marquée en gras. 56. En comparant les traductions du tchèque en français et vice versa, il faut prendre en considération aussi la différence de taille entre les deux sous-corpus. En outre, les comparaisons des fréquences générales ne sont pas capables de repérer d'éventuels ajouts de certains signes de ponctuation compensant des omissions (dans nos analyses des 4000 occurrences concrètes de non1 :1 segments, ces cas étaient plutôt exceptionnels).

\section{RÉSUMÉS}

L'objectif de cet article est de présenter certains problèmes méthodologiques liés à l'exploitation de corpus parallèles, c'est-à-dire de corpus composés de textes originaux et de leurs traductions respectives, et de proposer des principes et des règles d'utilisation qui pourraient permettre de les résoudre. Sont traités successivement les facteurs liés à la taille et à la composition de corpus 
parallèles (section 2), les facteurs techniques tels que l'alignement ou l'annotation morphosyntaxique bilingue (section 3), la question épineuse de l'équivalence des versions parallèles (4.1), l'importance des métadonnées portant sur les originaux et sur les traductions (4.2) et les spécificités de la langue de traduction (4.3). Les différents principes méthodologiques sont illustrés par des recherches concrètes réalisées sur le corpus parallèle InterCorp.

In this article, we present methodological problems pertaining to the exploitation of parallel corpora, i.e. corpora composed of translations and their respective originals, and we try to propose the principles and rules helping to avoid said problems. We treat successively factors of the size and composition of parallel corpora (section 2), technical factors such as alignment or bilingual POS-tagging (section 3), the tricky question of the equivalence of the parallel versions (4.1), the importance of metadata about the originals as well as the translations (4.2) and the particularities of the language of translation (4.3). The methodological principles are illustrated by specific studies on the parallel corpus InterCorp.

\section{INDEX}

Keywords : parallel corpus, methodology, contrastive linguistics, translation, punctuation, gerund

Mots-clés : corpus parallèle, méthodologie, linguistique contrastive, traduction, ponctuation, gérondif

\section{AUTEUR}

\section{OLGA NÁDVORNÍKOVÁ}

Université Charles de Prague 